\title{
The role of geometrically necessary dislocations in giving material strengthening
}

\author{
N.A. Fleck ${ }^{\mathrm{a}, *}$, M.F. Ashby ${ }^{\mathrm{a}}$, J.W. Hutchinson ${ }^{\mathrm{b}}$ \\ a Department of Engineering, Cambridge University, Trumpington Street, Cambridge, CB2 1PZ, UK \\ ${ }^{\mathrm{b}}$ Division of Engineering and Applied Sciences, Harvard University, Oxford Street, Cambridge, MA 02139, USA
}

Accepted 9 August 2002

\begin{abstract}
The role of geometrically necessary dislocations in providing macroscopic strengthening is reviewed with particle reinforcement as an example. The general relation between the Nye tensor and slip along a curvilinear slip line net is derived to emphasise the importance of curvature of slip lines upon the rate of dislocation storage.
\end{abstract}

(C) 2002 Acta Materialia Inc. Published by Elsevier Science Ltd. All rights reserved.

Keywords: Dislocation; Micromechanical modelling; Plastic deformation

\section{Introduction}

\subsection{Relation between dislocation density and slip}

Nye [1] has shown that the density of geometrically necessary dislocations may be quantified by a second rank tensor $\boldsymbol{\alpha}$. In order to calculate the density of geometrically necessary dislocations, a crystal lattice must be embedded within the solid: it is insufficient to know only the plastic strain distribution. For subsequent discussion, we summarise the relation between the Nye tensor and crystallographic slip from Fleck et al. [2]. We assume that the material shears through the crystal lattice by dislocation motion, and that the lattice (and attached material) undergoes rotation and

\footnotetext{
${ }^{*}$ Corresponding author. Tel.: +44-1223332650; fax: +441223332662.

E-mail address: naf1@eng.cam.ac.uk (N.A. Fleck).
}

elastic stretching. With $v_{i}$ as the velocity at a material point $x_{j}$, the velocity gradient $v_{i, j}$ can be written as the sum of a slip rate tensor $\dot{\gamma}_{i j}$, a lattice rotation rate tensor $\dot{\phi}_{i j}$ and the elastic strain rate tensor for the lattice $\dot{\varepsilon}_{i j}^{\mathrm{EL}}$, giving

$v_{i, j}=\dot{\gamma}_{i j}+\dot{\phi}_{i j}+\dot{\varepsilon}_{i j}^{\mathrm{EL}}$

The lattice rotation rate is anti-symmetric and can be expressed equivalently in terms of the lattice rotation rate vector $\omega_{i}$ where

$\omega_{q} \equiv \frac{1}{2} e_{j i q} \dot{\phi}_{i j}$

while the elastic strain rate $\dot{\varepsilon}_{i j}^{\mathrm{EL}}$ is symmetric.

A particular slip system, $\beta$, is specified by the vectors $\left(\boldsymbol{s}^{(\beta)}, \boldsymbol{m}^{(\beta)}\right)$ where $\boldsymbol{s}^{(\beta)}$ is the slip direction and $\boldsymbol{m}^{(\beta)}$ is the slip plane normal. Then, the slip rate tensor $\dot{\gamma}_{i j}$ is the net slip rate due to a slip $\dot{\gamma}^{(\beta)}$ on each of the slip systems, 
$\dot{\gamma}_{i j}=\sum_{\alpha} \dot{\gamma}^{(\beta)} s_{i}^{(\beta)} m_{j}^{(\beta)}$

with the summation taken over all slip systems. The density of geometrically necessary dislocations is related to the Burger's vector $B_{i}$ associated with crystallographic slip. Make an imaginary cut in the crystal in order to produce a surface $S$ of outward normal $\boldsymbol{n}$. Define $\dot{B}_{i}$ as the displacement discontinuity rate due to slip on completion of a Burger's circuit around the periphery $\Gamma$ of the surface S. In other words, $\dot{B}_{i}$ completes the circuit when $\Gamma$ is traversed in the sense of a right-handed screw motion along $\boldsymbol{n}$. Thus, $\dot{B}_{i}$ is

$\dot{B}_{i}=\int_{\Gamma} \dot{\gamma}_{i j} \mathrm{~d} x_{j}$

and can be rewritten using Stokes' theorem as

$\dot{B}_{i}=\int_{S} \dot{\alpha}_{i n} n_{n} \mathrm{~d} S$

where

$\dot{\alpha}_{i n}=e_{n k j} \dot{\gamma}_{i j, k}$

with $e_{n k j}$ denoting the alternating tensor. The tensor $\alpha$ is Nye's dislocation density tensor or torsionflexure tensor. It gives a direct measure of the number of geometrically necessary dislocations. Thus, geometrically necessary dislocations are associated with gradients of slip, and their density can be calculated directly by geometry once the active slip systems have been defined. The same displacement field may or may not give rise to geometrically necessary dislocations, depending upon the distribution of active slip systems. Lattice rotation (and elastic stretching of the lattice) is usually needed in order to ensure compatibility of displacement.

\section{Strengthening mechanisms due to geometrically necessary dislocations}

The precise manner by which geometrically necessary dislocations provide macroscopic strengthening remains elusive. Hardening is by local dislocation interaction mechanisms such as jog formation when a moving dislocation cuts through an existing geometrically necessary dislocation (and vice versa), and may be enhanced by the build-up of long range back stresses. The former mechanism provides macroscopic isotropic hardening, while the latter mechanism gives kinematic hardening. The relative importance of each hardening mechanism varies from problem to problem, and an example now follows where the relative significance varies with level of overall imposed strain and structural size.

\section{Case study: the distribution of dislocations around elastic particles in a dispersion strengthened alloy}

No simple relation exists between the density of geometrically necessary dislocations and the degree of kinematic versus isotropic hardening. It is illustrated below that certain observed distributions of dislocation density give rise to long range elastic stresses and to kinematic hardening, while other observed distributions give rise to isotropic hardening. For the purposes of discussion we consider the well-documented case of a dispersion strengthened alloy [3,4].

The physical picture of dislocation distribution around each non-deformable spherical particle is dependent upon the diameter of the particle and upon the overall level of plastic strain. Consider first, the case of small particles, of diameter less than about $150 \mathrm{~nm}$. At low levels of overall plastic shear strain (less than a few percent), geometrically necessary dislocations accumulate in the form of Orowan loops, giving rise to long range elastic back stresses and to strong kinematic hardening. The resolved shear stress on the slip planes equals the (low) Peierls stress, with a substantial fraction of the macroscopic stress carried by the elastic particle. A suitable continuum idealisation is that the elastic particle is surrounded by an external penny-shaped mode II crack. Large elastic stresses are generated in the vicinity of each particle, and the macroscopic shear response has a tangent modulus which scales with the volume fraction of particles.

Second, consider the case of small particles (of diameter less than about $150 \mathrm{~nm}$ ) within a single crystal subjected to macroscopic shear strains of 
above a few percent. Stacks of prismatic dislocation loops are punched into the crystal by the elastic particle; these loop stacks contain dislocations of the same primary Burgers vector as those responsible for plastic flow of the matrix. The loop stacks induce no lattice rotation, and give rise to negligible long range back stresses and to negligible kinematic hardening.

Third, rotational structures form at strains of above a few percent around particles of diameter exceeding about $150 \mathrm{~nm}$. The plastic strain state associated with these rotational structures is closely related to the centred fan of a slip line field, and a scheme is developed below for calculating the associated density of geometrically necessary dislocations. The rotational structure gives no long range back stress and persists to large levels of plastic strain.

In summary, the dominant source of hardening (kinematic hardening in nature) at levels of plastic strain below a few percent is by the generation of long range elastic stresses generated by a gradient of $\boldsymbol{\alpha}$. These long range stresses are relaxed by crossslip and secondary slip at higher levels of plastic strain, and the dominant source of hardening becomes isotropic hardening associated with shortrange dislocations interactions. The level of hardening scales with the density of geometrically necessary dislocations $\boldsymbol{\alpha}$.

\section{Calculation of dislocation density from a slip line field solution}

Slip line field solutions provide much insight into the state of fully developed plastic flow within an isotropic ideally plastic continuum. With suitable approximations, a slip line field solution can also be used to estimate the local density of geometrically dislocations within a crystal. The slip line field solution for a rigid, ideally plastic solid provides useful insight into the distribution of plastic strain for a plane strain deformation field deep in the plastic range. In order to estimate the slip distribution, an arbitrary choice is required for the active slip systems, and here we assume that the crystal has an infinite number of slip systems available over all orientations. (The accuracy of this simplification will depend upon the actual number of slip systems available in any given crystal). The crystal is taken as rigid, ideally plastic, and at any point in the deforming solid two slip systems aligned with the local slip lines are taken as potentially active. The slip line field solution provides the velocity gradient, but an additional argument is required in order to apportion the velocity gradient between the slip on each of the two available slip systems. This is made clear below for the case of the Prandtl field.

\section{Dislocation storage along curved slip lines}

Consider a curvilinear slip-line field, as sketched in Fig. 1. The distance along the $\alpha$-slip lines is measured by the co-ordinate $\xi$ while the distance along the orthogonal $\beta$-slip lines is given by $\eta$. The out-of-plane dimension is labelled $x_{3}$, and the curvature of the $\alpha$-slip lines is denoted by $\kappa_{\xi}$ while the curvature of the $\alpha$-slip lines is denoted by $\kappa_{\beta}$. Suppose that the slip rate along the $\alpha$-slip lines is $f(\xi, \eta)$ while the slip rate along the $\beta$-slip lines is $g(\xi, \eta)$. Then, the rate of accumulation of the Nye tensor follows from the prescription (6), which gives after some manipulation,

$\dot{\alpha}_{\xi 3}=\frac{\partial f}{\partial \xi}+(f-g) \kappa_{\eta}$

$\dot{\alpha}_{\eta 3}=\frac{\partial g}{\partial \eta}+(f-g) \kappa_{\xi}$

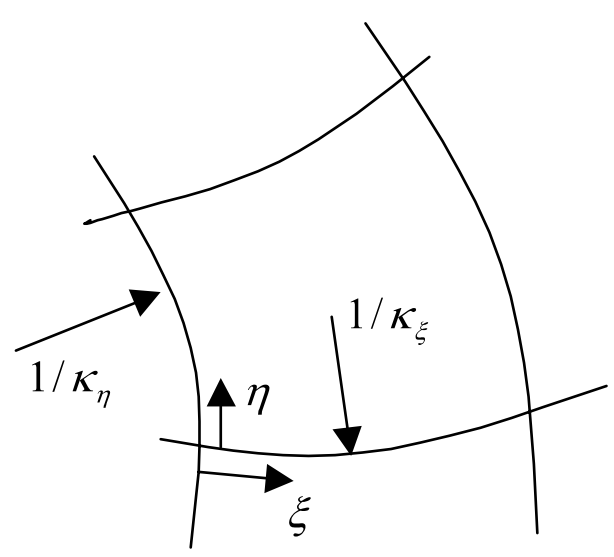

Fig. 1. Curvilinear slip line field. 
We conclude that dislocation storage occurs when a gradient of slip occurs along a slip line, and when slip occurs along a curved slip line.

\section{Dislocation storage for the Prandtl field}

The Prandtl slip line field [5], as sketched in Fig. 2 , provides an illustration of the use of relation (7a) and (7b) in order to estimate the density of geometrically necessary dislocations. Consider the advance of a frictionless flat punch into a rigid, ideally plastic solid, at a normal velocity $V$. Then, the deformation state within the indented solid is given by the Prandtl slip-line field, comprising rigid triangles and centred fans.

Take a polar co-ordinate system $(r, \theta)$ centred at the apex of each fan. Then, within each centred fan, the velocity gradient can be re-written in terms of the rigid body rotation rate $\omega$, the slip rate $f(r)$ along the radial slip lines, and the slip rate $g(r)$ along the circumferential slip lines as

$f-\omega=-\frac{V}{r \sqrt{2}}$

and

$\omega-g=0$

It is clear that an additional assumption is required in order to deduce the value of $(f, g, \omega)$ from the two equations (8a) and (8b). It is argued on physical grounds that the presence of the rigid substrate prevents slip along the radial direction

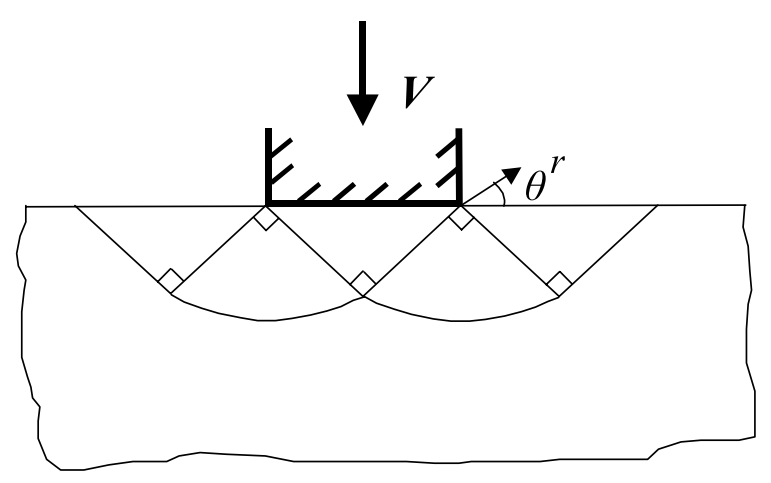

Fig. 2. The Prandtl indentation field. within each centred fan-in the actual dislocated solid, dislocation pile-ups accumulated along the radial slip planes and give rise to back stresses which switch off radial slip, giving $f \equiv 0$. Consequently, relations (8a) and (8b) reduce to

$g=\omega=\frac{V}{r \sqrt{2}}$

and the rate of accumulation of dislocation density within each centred fan follows from (7b) as

$\dot{\alpha}_{r 3}=-\frac{V}{r^{2} \sqrt{2}}$

We conclude that slip along the circular arcs of each centred fan gives rise to the storage of geometrically necessary dislocations, and hence to work hardening. The ambiguity in relative amount of slip on the two potentially active systems is resolved by arguing that slip along the radial lines within the centred fan fields will give rise to dislocation pile-ups against the adjacent rigid regions, and thereby the radial slip system will switch off due to the build up of back stress.

Alternative approaches may be taken to remove the ambiguity of slip: it could be argued that the crystal adopts a distribution of slip which minimises the elastic free energy associated with the dislocation density, $\boldsymbol{\alpha}$. But this assumption would imply that the radial slip system is activated (it generates no contribution to $\alpha$ ) while the circumferential slip system is switched off. Or we could adopt Havner's postulate of minimum relative spin [6]: the active slip systems are arranged such that the difference between the material spin rate and the lattice spin rate is minimised. This would imply that the amount of $\operatorname{slip} f$ on the radial slip planes equals the amount of slip $g$ on the circumferential slip planes. Neither of these approaches is in agreement with the physical argument that $f=0$.

\section{Acknowledgements}

The authors are grateful for helpful discussions with L.M. Brown, V.S. Deshpande and A. Needleman. 


\section{References}

[1] Nye JF. Acta Metall 1953;1:153.

[2] Fleck NA, Muller GM, Ashby MF, Hutchinson JW. Acta Metall Mater 1994;42:475.

[3] Brown LM. Phil Trans Royal Soc Lond A 1997;355:1979.
[4] Ashby MF. Phil Magazine 1970;21:399.

[5] Hill R. The mathematical theory of plasticity. Oxford: Oxford University Press; 1950.

[6] Havner KS. Finite plastic deformation of crystalline solids. Cambridge: Cambridge University Press; 1992. 